\title{
Much Ado About Nipples
}

\author{
Suzanne B. Coopey, $\mathrm{MD}^{1}$ \\ Division of Surgical Oncology, Massachusetts General Hospital, Harvard Medical School, Boston, MA
}

The nipple areola complex (NAC) is the focal point of the breast. Saving the NAC at the time of mastectomy provides women with a postoperative reconstructed breast appearance that is most like their native breast. Many women who require or elect to have a mastectomy prefer to keep their nipple(s). When nipples are spared at the time of mastectomy plus reconstruction, postoperative psychosocial well-being and sexual well-being are significantly better. $^{1}$

Despite increased utilization of nipple-sparing mastectomy (NSM) for breast cancer treatment over the past decade, some remain concerned about its oncologic safety given the lack of long-term follow-up data. Sites potentially at risk for new or recurrent breast cancer after NSM include the retained NAC and the mastectomy skin flaps, particularly at the periphery of the breast where visualization may be more difficult, with the inframammary or inferolateral incisions typically used for NSM. Successful NSM requires careful excision and pathologic evaluation of the nipple ductal tissue, as well as thorough removal of all visible breast tissue, much like any mastectomy. Because there are no randomized controlled trials comparing NSM with skin-sparing mastectomy or simple mastectomy, continued publication of NSM outcomes data is necessary to confirm the safety of this technique.

In this issue of Annals of Surgical Oncology, Valero and colleagues review the outcomes of 449 women with breast cancer who underwent $770 \mathrm{NSMs}$ at Memorial Sloan Kettering Cancer Center (MSKCC) from 2003 to $2016 .{ }^{2}$ Of 467 therapeutic NSMs, $337(72.2 \%)$ were for invasive

(C) Society of Surgical Oncology 2019

First Received: 14 September 2019; Published Online: 18 October 2019

S. B. Coopey, MD

e-mail: scoopey@mgh.harvard.edu cancer, $126(27.0 \%)$ were for ductal carcinoma in situ (DCIS), and $4(0.9 \%)$ were for phyllodes tumors. They excluded patients with locally advanced disease, disease $\leq 1 \mathrm{~cm}$ from the nipple, direct nipple involvement, and those with extensive disease in the periphery of the breast (due to concerns about limited exposure). This last exclusion is unusual as careful peripheral dissection is important for any mastectomy and can be equally challenging for a skin-sparing mastectomy through a small periareolar incision. They did not exclude patients based on unfavorable histological subtypes. In fact, $16.1 \%$ of invasive cancers were human epidermal growth factor receptor 2 (HER2)positive and $10.2 \%$ were triple-negative. With careful patient selection based on the above criteria, only 14 of 467 (3.0\%) therapeutic NSMs in their cohort required nipple excision due to positive nipple margins.

At a median follow-up of 39.4 months, Valero and colleagues report only four total locoregional recurrences (LRRs) in $449(0.9 \%)$ patients. $^{2}$ None of the LRRs involved the retained NAC. No new second primary breast cancers were reported, despite inclusion of 46 patients who were positive for BRCA1 or BRCA2 mutations, lending further evidence to the safety of NSM even in mutation carriers. There were 11 isolated distant metastases and 1 LRR plus distant metastasis during the follow-up period, suggesting that systemic risk of recurrence is of greater concern than LRR. Similarly, when Peled et al. ${ }^{3}$ reviewed the outcomes of 139 patients with locally advanced stage IIB or III breast cancer treated with NSM, they concluded that NSM was not associated with an increased risk of local recurrence and that the real risk was for distant spread.

The article by Valero et al. on the increase in utilization of NSM for breast cancer does not specify which types of incisions were used for NSM at MSKCC. While an inframammary incision provides an entirely hidden scar along the fold of the breast, it can make it difficult to visualize the superior pole of the breast. This is highlighted in a study of 518 NSMs for breast cancer by Tomasi Cont et al., ${ }^{4}$ who 
discovered that most local recurrences after NSM occurred in the superior half of the reconstructed breast and typically in the location of the primary tumor. The inferolateral incision, which is placed along the lateral half of the fold and curved up along the lateral edge of the breast, provides easier access to the axilla and upper outer breast than an inframammary incision, but the upper inner breast dissection can still be challenging. ${ }^{5}$ For this reason, it is always important to check the peripheral skin flap thickness after the breast is removed to ensure all visible breast tissue has been adequately excised. If a patient's tumor is known to be peripherally located, a mastectomy specimen radiograph can be useful to verify tumor removal intraoperatively. In extreme cases, cancers can also be localized with image guidance prior to performing NSM to ensure removal of the intended target.

The authors' preferred method for obtaining a nipple margin specimen during NSM is not described in the paper by Valero et al. However, in their Introduction, there is mention of leaving a small amount of ductal tissue beneath the nipple. ${ }^{2}$ While this was common practice with the older subcutaneous mastectomy technique for risk reduction, with the more modern NSM technique it is preferable to remove all glandular tissue beneath the NAC. A practice adopted by our group at Massachusetts General Hospital, based on our microanatomy studies, involves raising areola skin flaps, but leaving the nipple duct bundle intact, then grasping the duct bundle with a curved clamp immediately below the NAC dermis, sharply dividing the tissue along both sides of the clamp, and sending the contents of this clamp as the nipple margin specimen. ${ }^{6-8}$ This approach completely clears the nipple-areola skin of fat, breast parenchyma, and ductal tissue. Doing this eliminates the need for additional sharp dissection within the nipple papilla and results in very low rates of nipple loss due to necrosis ( $1.7 \%$ overall, $0.9 \%$ without radiation), despite not leaving any glandular tissue beneath the nipple. ${ }^{9,10}$ It also ensures that the nipple ducts do not retract into the mastectomy specimen, which could yield an inadequate nipple margin specimen. ${ }^{7}$

Our practice considers NSM the default procedure for anyone having reconstruction, with many more exceptions for cosmetic considerations rather than for concerns about oncologic safety. NSM is a technically demanding operation requiring meticulous dissection and careful flap retraction to optimize oncologic safety and minimize complications. Breast surgeons have risen to the challenge and NSM has become a standard part of the repertoire of most breast surgeons and training programs. The article by Valero and colleagues adds to the growing body of evidence in support of NSM in the majority of women with breast cancer.

\section{REFERENCES}

1. Romanoff A, Zabor EC, Stempel M, Sacchini V, Pusic A, Morrow MA. A comparison of patient-reported outcomes after nipple-sparing mastectomy and conventional mastectomy with reconstruction. Ann Surg Oncol. 2018;25:2909-16.

2. Valero MG, Muhsen S, Moo T-A, et al. Increase in utilization of nipple-sparing mastectomy for breast cancer: indications, complications, and oncologic outcomes. Ann Surg Oncol. 2019. h ttps://doi.org/10.1245/s10434-019-07948-x.

3. Peled AW, Wang F, Foster RD, Alvarado M, Ewing CA, Sbitany $\mathrm{H}$, et al. Expanding the indications for total skin-sparing mastectomy: is it safe for patients with locally advanced disease? Ann Surg Oncol. 2016;23:87-91.

4. Tomasi Cont N, Maggiorotto F, Martincich L, et al. Primary tumor location predicts the site of local relapse after nippleareola complex (NAC) sparing mastectomy. Breast Cancer Res Treat. 2017;165:85-95.

5. Colwell AS, Gadd M, Smith BL, Austen WG. An inferolateral approach to nipple-sparing mastectomy optimizing mastectomy and reconstruction. Ann Plast Surg. 2010;65:140-3.

6. Rusby JE, Brachtel EF, Michaelson JS, Koerner FC, Smith, BL. Breast duct anatomy in the human nipple: three dimensional patterns and clinical implications. Breast Cancer Res Treat. 2007;106:171-9.

7. Rusby JE, Kirstein LJ, Brachtel EF, et al. Nipple-sparing mastectomy: lessons from ex vivo procedures. Breast $J$. 2008; 14:464-70

8. Coopey SB, Smith BL. The nipple is just another margin. Ann Surg Oncol. 2015;22:3764-6.

9. Coopey SB, Tang R, Lei L, et al. Increasing eligibility for nipple sparing mastectomy. Ann Surg Oncol. 2013;20:3218-22.

10. Tang R, Coopey SB, Colwell AS, et al. Nipple-sparing mastectomy in irradiated breasts: selecting patients to minimize complications. Ann Surg Oncol. 2015;22:3331-7.

Publisher's Note Springer Nature remains neutral with regard to jurisdictional claims in published maps and institutional affiliations. 